\title{
Semiconducting Metal Oxide Nanoarchitectures: Harnessing Solar Energy for Electricity and Hydrogen Production
}

Jingyue (Jimmy) Liu

Center for Nanoscience, Dept of Physics \& Astronomy, and Dept of Chemistry \& Biochemistry, University of Missouri-St. Louis, One University Blvd., St. Louis, MO 63121, USA (liuj@umsl.edu)

The ever increasing energy demand of a growing population on our planet requires development of environmentally clean and sustainable energy resources. In addition to its cleanness, the enormous potential of sunlight (more energy from sunlight strikes the earth per hour than all of the energy consumed on the planet in one year) is a major driving force for developing economically feasible technologies to harness sunlight as an alternative energy resource. Photovoltaic conversion, the direct generation of electricity from sunlight, is a clean and inexhaustible source of electricity generation. Similarly, hydrogen production, in a photoelectrochemical (PEC) cell, by catalystfacilitated water splitting, is a desirable clean process for converting solar energy and storing it in hydrogen. The challenge to broad utilization of solar energy is that major technological breakthroughs have to be achieved to make it economically competitive with conventional, albeit not environmentally friendly, energy resources such as fossil fuels. The emergence of nanomaterials, with their unique properties, as the new building blocks to construct light-harvesting, conversion and storage devices has opened the door to developing efficient and inexpensive solar cells [1-3]. Nanostructured metal oxides offer great potential for developing efficient dye sensitized solar cells (DSSC) [1-3] and are excellent candidates as photoelectrodes in PEC cells [4-5].

The fundamental material requirements for constructing high efficiency DSSC and PEC cells have common features: strong absorption in the solar spectrum region, fast interfacial electron transfer, low rate of electron-hole recombination, high electron mobility, resistance to photo/photochemical degradation, and low cost. The use of nanowire arrays to construct DSSCs has great potential because of the direct electrical pathways (to the anode) provided by the nanowires, ensuring rapid collection of carriers generated throughout the device [2]. The loading of dyes to nanowire arrays is a critical factor that determines the efficiency of nanowire-based DSSCs. High-surface area of electrodes is needed for both DSSCs and PEC cells. We have been focusing on developing highsurface area, single-crystal metal oxide nanostructures such as dense $\mathrm{ZnO}$ nanowires (Fig.1) and hierarchical $\mathrm{ZnO}$ nanobelts (Fig. 2) for DSSCs, $\mathrm{Fe}_{2} \mathrm{O}_{3}$ nanobelts/nanowires as photoanodes for water splitting, $\mathrm{Cu}_{2} \mathrm{O} / \mathrm{CuO}$ nanowires/nanobelts for hydrogen production, and composites of these nanostructures to improve their conversion efficiencies in DSSCs and to make them stable under photoelectrochemical conditions.

Figure 1 shows a SEM image of densely populated and aligned ZnO nanowire arrays. Such nanowire arrays can be further treated to significantly increase the total available surface area, thus increasing the loading of sensitizers to increase conversion efficiency. Figure 2 shows a SEM image of hierarchical $\mathrm{ZnO}$ nanoarchitectures to increase the dye loading and to enhance the absorption of sunlight by scattering. Figure 3a shows a low magnification SEM image of "trees" of $\mathrm{ZnO}$ nanostructures and Fig. $3 \mathrm{~b}$ shows the dense packing of very thin $\mathrm{ZnO}$ nanowires epitactically grown on the "tree stem", thus maintaining the properties of a single crystal. Porous, doped and nanocomposite metal oxide nanostructures have been developed. Advanced electron microscopy and $\mathrm{X}$-ray scattering characterization of hierarchical $\mathrm{ZnO}$ nanoarchitectures, $\mathrm{Fe}_{2} \mathrm{O}_{3}$ nanobelts/nanowires, $\mathrm{CuO}$ nanowires/nanobelts, and composites of these nanostructures will be discussed [6]. 


\section{References}

[1] B. O'Regan and M. Grätzel, Nature 353 (1991) 737.

[2] M. Law et al., Nature Materials 4 (2005) 455.

[3] L. Tsakalakos, Mater. Sci. \& Eng. R 62 (2008) 175.

[4] A. Kudo and Y. Miseki, Chem. Soc. Rev. 38 (2009) 253.

[5] P. V. Kamat, J. Phys. Chem. C 111 (2007) 2834.

[6] This research was supported by the University of Missouri-St. Louis.
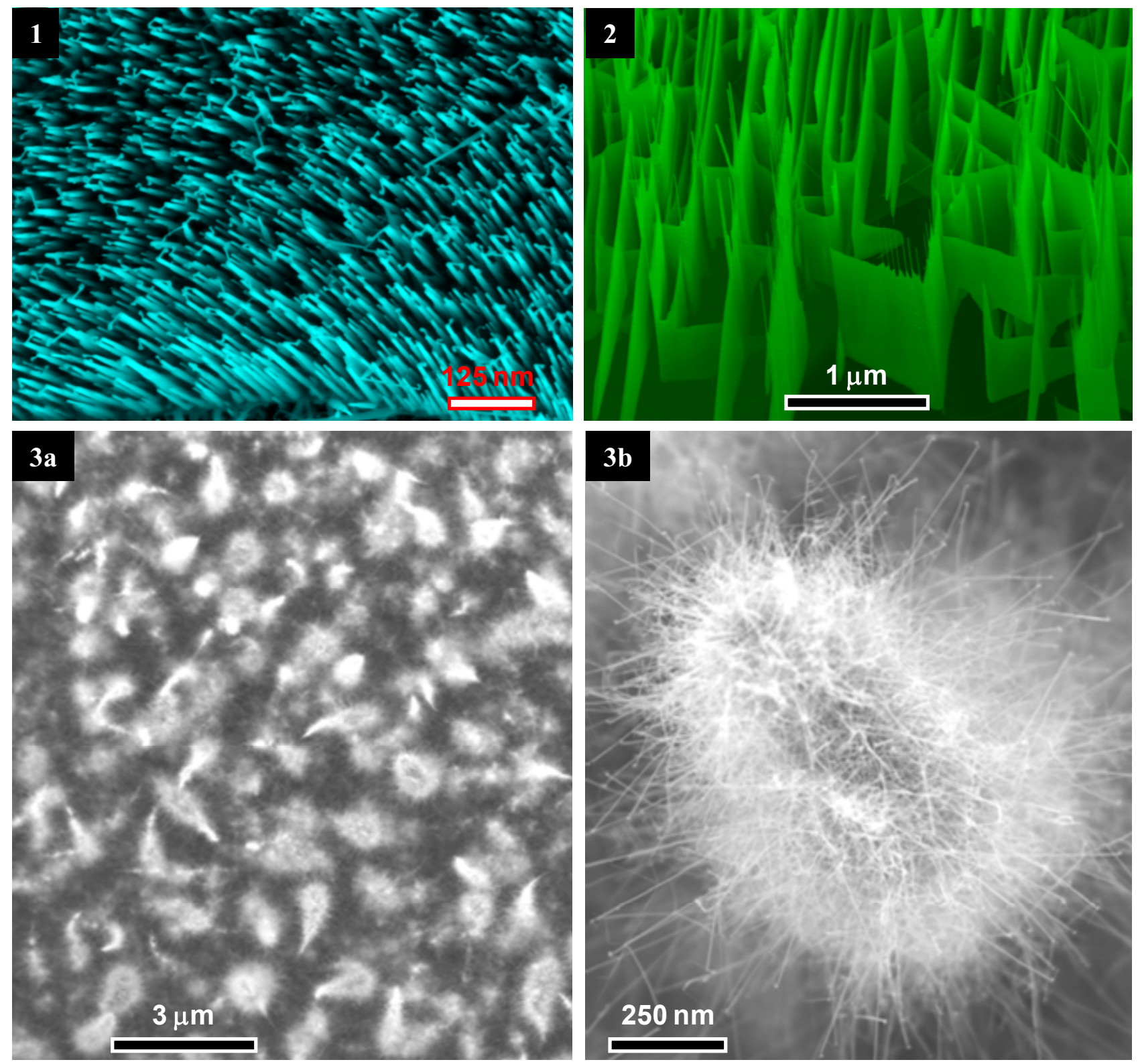

Fig. 1. SEM image shows dense, aligned $\mathrm{ZnO}$ nanowires for applications in solar cells.

Fig. 2. SEM image shows high surface area, hierarchical $\mathrm{ZnO}$ nanoshelves for enhanced lightharvesting and catalytic performance.

Fig. 3. Low magnification (a) and high magnification (b) SEM images show "trees" with highdensity nanowires grown epitactically onto the "tree stems". 